\title{
First Report of Meloidogyne haplanaria Infecting Mi-Resistant Tomato Plants in Florida and Its Molecular Diagnosis Based on Mitochondrial Haplotype
}

\author{
Soumi Joseph and Tesfamariam Mekete, University of Florida, Entomology and Nematology Department; Gainesville 32611; Wiseborn B. \\ Danquah, Department of Plant Pathology, University of California, Davis 95616; and Joseph Noling, University of Florida, Institute of Food \\ and Agricultural Sciences, Citrus Research \& Education Center, Lake Alfred 33850
}

\begin{abstract}
Joseph, S., Mekete, T., Danquah, W. B., and Noling, J. 2016. First report of Meloidogyne haplanaria infecting Mi-resistant tomato plants in Florida and its molecular diagnosis based on mitochondrial haplotype. Plant Dis. 100:1438-1445.

Meloidogyne haplanaria is a species originally found infesting peanut in Texas and, more recently, in Arkansas. In this study, we confirmed the presence of M. haplanaria in Florida based on morphological and molecular characterization. This species was identified from a sample submitted for diagnosis collected from $\mathrm{Mi}$-resistant tomato rootstock grown in Naples, FL. The major diagnostic criteria to distinguish $M$. haplanaria from other closely related root-knot nematode (RKN) species are based on morphological differences and host range tests, which are time consuming and labor intensive and require living or well-preserved specimens. In our study, we provide an easy diagnostic strategy to distinguish

M. haplanaria from other RKN species based on amplification of two mitochondrial DNA regions. These regions span the intergenic spacer and part of the adjacent large subunit ribosomal RNA gene (lrDNA) and sequence polymorphisms in lrDNA revealed by the restriction pattern following digestion with the restriction enzymes HinfI and MnII A unique haplotype pattern, which has not been observed in any of the RKN species described thus far, was observed in M. haplanaria. The outcome of molecular analysis of $M$. haplanaria aligned with morphological measurement and characteristics as well as perineal pattern originally described for M. haplanaria.
\end{abstract}

Meloidogyne spp. (root-knot nematodes [RKN]) are economically important plant parasites with over 5,500 plant hosts (Trudgill and Blok 2001). Solanaceous plants such as tomato, potato, eggplant, and pepper are among the main hosts for RKN. Meloidogyne haplanaria, the Texas peanut RKN, was originally found attacking peanut in Texas. The current known distribution of M. haplanaria is limited to peanut in Texas (Eisenback et al. 2003) and, according to a recent report, Arkansas (Churamani et al. 2015). M. haplanaria has yet to be reported in Florida. Host range studies revealed that $M$. haplanaria can parasitize several legumes and crucifer crops (Eisenback et al. 2003) and has also been shown to infect $M$. arenaria-susceptible cultivars of peanut, garden pea, and radish (Bendezu et al. 2004). This host range is similar to several other RKN in that it is likely to successfully parasitize a large and diverse number of hosts across a large range of plant families (Bendezu et al. 2004). Although watermelon, cotton, maize, tobacco, and wheat are nonhosts for $M$. haplanaria, pepper, eggplant, soybean, and common bean are moderate hosts for this nematode (Bendezu et al. 2004; Eisenback et al. 2003).

The peanut 'NemaTAM', which has been bred for resistance to M. arenaria and M. javanica (Simpson et al. 2003), is also resistant to $M$. haplanaria; however, the resistance in tomato to $M$. arenaria, $M$. javanica, and M. incognita conferred by the Mi gene (Williamson 1998) is not effective against $M$. haplanaria (Bendezu et al. 2004). Identifying the presence of this nematode and mitigating its impact in Florida is essential because Florida is one of the leading peanut producing states in the United States (FDACS 2012). Additionally, it is the second largest producer of vegetables such as tomato, bell pepper, snap bean, squash, watermelon, cabbage, and cucumber in the United States, with cash receipts of $\$ 1.93$ billion (13\% of the total U.S. value; FDACS 2012). M. haplanaria has been shown to overcome the resistance in tomato conferred by the $M i$ gene (Bendezu et al. 2004). Currently, resistance to RKN present in commercial

Corresponding author: J. Soumi; E-mail: sjoseph80@ufl.edu

Accepted for publication 10 February 2016

http://dx.doi.org/10.1094/PDIS-09-15-1113-RE

(C) 2016 The American Phytopathological Society varieties of tomato is conferred by this gene; therefore, it is important to assess the potential for the establishment of this resistancebreaking RKN in tomato- and peanut-growing areas in Florida. In August 2014, the occurrences of RKN on resistant tomato cultivars grown in Naples, FL came to our attention through the Nematode Laboratory of the University of Florida Institute of Food and Agricultural Sciences (UF/IFAS). Typical RKN symptoms, such as stunting and extensive root galls, were found on the roots of these tomato plants.

Detailed molecular and morphological studies of the nematode samples supported the conclusion that the species of RKN occurring in the fields in Naples, FL were different from the major groups of nematodes known to be present in the region and were also different from $M i$ resistance-breaking species such as M. hapla and M. enterolobii. The objective of this study was to identify the Meloidogyne spp. found in Naples, FL by morphological and molecular methods. Eisenback and colleagues (Eisenback 1985; Eisenback et al. 2003) provided a diagnostic key for distinguishing the most common species of RKN (M. arenaria, M. hapla, $M$. incognita, and $M$. javanica) and M. haplanaria based on morphological differences and host range tests. However, these approaches are time consuming and labor intensive and require living or wellpreserved specimens. DNA-based identification using primers that amplify a specific genomic fragment from a target species has been useful for several RKN species (Adam et al. 2007; Blok and Powers 2009). However, this approach has limited application because a negative result could indicate either a failed reaction or an indication that the nematode is a different species or variant. Although the DNA sequences of ribosomal RNA (rDNA) genes have been used as diagnostic tools for many organisms, this approach is limited in differentiating RKN species because some species (particularly $M$. incognita, $M$. arenaria, and $M$. javanica) show greater diversity in sequence of rDNA copies within an individual than between species (Pagan et al. 2015). In contrast to this, the mitochondrial genome of apomictic RKN species, due to its uniparental inheritance, has been shown to be a useful source of diagnostic markers (Powers et al. 1986). Recent studies (Kiewnick et al. 2014; Pagan et al. 2015) have demonstrated that two typical DNA barcoding genes, the mitochondrial cytochrome $\mathrm{c}$ oxidase subunit I (COI) and mitochondrial cytochrome c oxidase subunit II (COII), are probably be the best molecular markers to discriminate closely related RKN species. Polymorphisms in the length of the 
noncoding intergenic spacer (IGS) between COII and the large subunit of rDNA of the mitochondrial DNA (mtDNA), together with restriction fragment length polymorphisms of this region, have been used as reliable diagnostic marker to distinguish major RKN species (Powers and Harris 1993; Powers et al. 1986). Pagan et al. (2014) adopted a diagnostic strategy based on amplification of two mtDNA regions that, together, span the spacer and part of the adjacent large subunit (16S) rRNA (lrDNA) gene to characterize different RKN species by assigning a mitochondrial haplotype. However, to the best of our knowledge, a reliable molecular diagnostic tool to identify $M$. haplanaria has yet to be reported.

Due to the economic importance of $M$. haplanaria, accurate identification of RKN in Florida is highly essential to efficiently employ management strategies against this group of economically important nematodes. In our study, we provide an easy diagnostic strategy to distinguish $M$. haplanaria from other RKN species based on amplification of two mtDNA regions that, together, span the spacer and part of the adjacent lrDNA gene (Pagan et al. 2015). We assigned a mitochondrial haplotype of $M$. haplanaria based on the size of the IGS and sequence polymorphisms in the lrDNA following digestion with the restriction enzymes HinfI and MnlI. This study provides an easy approach to distinguish $M$. haplanaria from the major RKN species, including $M$. hapla and the highly pathogenic and invasive M. enterolobii.

\section{Materials and Methods}

Sample source and nematode isolation. In August 2014, heavily galled tomato roots and soil samples from a commercial vegetable field in Naples, FL were submitted to the UF/IFAS, Nematode Assay Laboratory (Gainesville, FL) for diagnosis and species identification. Second-stage juveniles (J2) were extracted from the soil samples using the sugar-floatation method (Harrison and Green 1976) and used for further molecular characterization. Ten singleegg masses (SEM) were extracted from the infected roots and propagated on tomato (Solanum lycopersicum L. 'Rutgers') grown in autoclaved sand in the greenhouse at $28^{\circ} \mathrm{C}$. After 8 weeks, gravid females were collected from the infected roots by manually dissecting the roots under a stereomicroscope followed by incubation in a 1:5 dilution of $100 \times$ Crystalzyme (White Laboratories, Davis, CA) pectinase solution for $2 \mathrm{~h}$.

Morphological characterization. Morphological and morphometric characteristics of $20 \mathrm{~J} 2$ hatched from SEM (Esser et al. 1976) collected from the greenhouse cultures were examined using live specimens relaxed with low heat (Esser 1986). Morphological characteristics were examined and compared with those reported in the original description of M. haplanaria by Eisenback et al. (2003) from Texas.

Perineal patterns of females were prepared in accordance with the method of Hartman and Sasser (1985). Ten Meloidogyne sp. females were dissected from the root galls of SEM lines (greenhouse cultured) and transferred to a drop of $45 \%$ lactic acid, and the perineal regions were cut and cleaned for microscopy. Morphological observations and photographs were completed within $24 \mathrm{~h}$ following slide preparation using a Leica DMLB compound microscope (Leica Microsystems, Buffalo Grove, IL) equipped with a JVC KY-F7OB digital camera (JVC, Wayne, NJ), and the images were processed using Auto-Montage Pr Software (version 5.02; Syncroscopy, Cambridge, UK).

Molecular characterization. DNA was extracted individually from eight gravid females collected from the greenhouse culture according to the procedure of Pagan et al. (2014). Briefly, a single female was transferred to $10 \mu \mathrm{l}$ of extraction buffer, consisting of $10 \mathrm{mM}$ Tris, $1 \mathrm{mM}$ EDTA, $0.1 \%$ triton $\mathrm{X}$, and proteinase $\mathrm{K}$ (polymerase chain reaction [PCR] grade; Fermentas, Grand Island, NY) at $1 \mathrm{mg} / \mathrm{ml}$, in a $1.5-\mathrm{ml}$ Eppendorf tube, and the nematodes were disrupted using a probe. Samples were frozen in 0.2-ml PCR tubes at $-20^{\circ} \mathrm{C}$ overnight. For DNA extraction, samples were incubated at $56^{\circ} \mathrm{C}$ for $1 \mathrm{~h}$ followed by $95^{\circ} \mathrm{C}$ for $10 \mathrm{~min}$, then used immediately for PCR or stored at $-20^{\circ} \mathrm{C}$. Additionally, DNA was extracted from 5 to $10 \mathrm{~J} 2$ extracted from the original field soil samples from Naples, FL. PCR amplification was performed on the extracted DNA using the RKN primers listed in Table 1. Amplification was carried out in $25 \mu \mathrm{l}$ of reaction mix containing $2 \times$ Taq Hot Start Master Mix (New England BioLabs, Ipswich, MA), $1.5 \mu \mathrm{l}$ of DNA, and $0.5 \mu \mathrm{M}$ each primer. The thermal cycling was performed in a Mastercycler nexus (Eppendorf AG, Hamburg, Germany). The thermocycling reactions using the primers TRNAH/MRH106 and MORF/MTHIS (Pagan et al. 2015; Stanton et al. 1997) were as follows: $95^{\circ} \mathrm{C}$ for $15 \mathrm{~min}$; followed by 35 cycles of $94^{\circ} \mathrm{C}$ for $1 \mathrm{~min}$, $50^{\circ} \mathrm{C}$ for $1 \mathrm{~min}$, and $68^{\circ} \mathrm{C}$ for $1 \mathrm{~min}$; and a final extension step of $68^{\circ} \mathrm{C}$ for $10 \mathrm{~min}$. The annealing temperature for other primer pairs was set as described previously (Table 1). DNA fragments were separated by electrophoresis in Tris-borate-EDTA buffer and $1.5 \%$ agarose gels. To determine the mitochondrial haplotype, the fragments

Table 1. Primers used for identification of Meloidogyne spp. and their sequences and sources

\begin{tabular}{|c|c|c|c|}
\hline Primer & Specificity & Sequence & Reference \\
\hline TRANAH & COII mtDNA region & TGAATTTTTTATTGTGATTAA & Stanton et al. (1997), Pagan et al. (2014) \\
\hline MRH106 & COII mtDNA region & AATTTCTAAAGACTTTTCTTAGT & Stanton et al. (1997), Pagan et al. (2014) \\
\hline MORF & lrDNA region & ATCGGGGTTTAATAATGGG & Stanton et al. (1997), Pagan et al. (2014) \\
\hline MTHIS & lrDNA region & AAATTCAATTGAAATTAATAGC & Stanton et al. (1997), Pagan et al. (2014) \\
\hline $\mathrm{C} 2 \mathrm{~F} 3$ & COII mtDNA region & GGTCAATGTTCAGAAATTTGTGG & Powers and Harris (1993) \\
\hline 1108 & Large subunit $16 \mathrm{SrRNA}$ & TACCTTTGACCAATCACGCT & Powers and Harris (1993) \\
\hline 194 & 5S-18S ribosome & TTAACTTGCCAGATCGGACG & Blok et al. (1997) \\
\hline 195 & 5S-18S ribosome & TCTAATGAGCCGTACGC & Blok et al. (1997) \\
\hline $63 \mathrm{VNL}$ & $63 \mathrm{bp}$ repeats of mtDNA & GAAATTGCTTTATTGTTACTAAG & Stanton et al. (1997) \\
\hline $63 \mathrm{VTH}$ & 63bp repeats of mtDNA & TAGCCACAGCAAAATAGTTTTC & Stanton et al. (1997) \\
\hline Me-F & Meloidogyne enterolobii specific & AACTTTTG TGAAAGTGCCGCTG & Hu et al. (2011) \\
\hline Me-R & M. enterolobii specific & TCAGTTCAGGCAGG ATCAACC- & Hu et al. (2011) \\
\hline JB3 & COI region of mtDNA & TTTTTTGGGCATCCTGAGGTTTAT & Kiewnick et al. (2014) \\
\hline CoI2R5 & $\mathrm{COI}$ region of mtDNA & YTRWYCTTAAATCTAAATKMGTATG & Kiewnick et al. (2014) \\
\hline Far & M. arenaria specific SCAR & TCGGCGATAGAGGTAAATGAC & Adam et al. (2007) \\
\hline Rar & M. arenaria specific SCAR & TCGGCGATAGACACTACAAACT & Adam et al. (2007) \\
\hline Fjav & M. javanica specific SCAR & GGTGCGCGATTGAACTGAGC & Zijlstra et al. (2000) \\
\hline \multirow[t]{2}{*}{ Rjav } & M. javanica specific SCAR & CAGGCCCTTCAGTGGAACTATAC & Zijlstra et al. (2000) \\
\hline & $\ldots$ & CAGGCCCTTCAGTGGAACTATAC & $\cdots$ \\
\hline MI-F & M. incognita specific SCAR & GTGAGGATTCAGCTCCCCAG & Meng et al. (2004) \\
\hline MI-R & M. incognita specific SCAR & ACGAGGAACATACTTCTCCGTCC & Meng et al. (2004) \\
\hline JMVI & M. hapla specific SCAR & GGATGGCGTGCTTTCAAC & Adam et al. (2007) \\
\hline JMV2 & M. hapla specific SCAR & TTTCСССТTATGATGTTTACCC & Adam et al. (2007) \\
\hline
\end{tabular}


amplified using the primer set TRNAH/MRH106 were subjected to restriction digestion using the restriction enzymes HinfI and MnlI (New England Biolabs), as recommended by the manufacturer.

The amplified PCR products of the mtDNA regions (COI and COII fragments) and IGS fragments were purified using either the QIAquick Gel purification kit (Qiagen, Valencia, CA) or the QIAquick PCR Purification kit (Qiagen), according to the manufacturer's instructions. The purified DNA was quantified using NanoDrop 1000 (Thermo Scientific, Grand Island, NY) spectrophotometer and ligated into the pDrive amp/Kan cloning vector kit, (Qiagen) according to the manufacturer's instructions. The ligation products were then transformed into recombinant cells (QIAGEN EZ Competent Cells) as per the manufacturer's instructions (Qiagen). The plasmids were extracted using the ZR plasmid miniprep kit (Zymo Research, Irvine, CA) and sequenced in both directions at the Interdisciplinary Center for Biotechnology Research, University of Florida, Gainesville. To identify the Meloidogyne spp., obtained raw sequences were checked and edited manually using BioEDIT, v. 7.0.9 (Hall 1999). Consensus sequences obtained were compared with those deposited in the GenBank database using a BLAST engine search for sequence homology (Benson et al. 2013).

Phylogenetic analysis. Unique sequences obtained for COI and COII gene regions in this study and those retrieved from the National

Table 2. Morphometrics of Meloidogyne haplanaria-second stage juveniles from Florida

\begin{tabular}{lcc}
\hline Characteristic & $\begin{array}{c}\text { Florida isolate } \\
(\boldsymbol{\mu m} \pm \mathbf{S E})^{\mathbf{a}}\end{array}$ & $\begin{array}{c}\text { Original } \\
\text { description }^{\mathbf{b}}\end{array}$ \\
\hline Body length & $448.2 \pm 2.3(435.2-470.5)$ & $419.0 \pm 3.9(365-480)$ \\
Body width & $19.0 \pm 0.2(16.2-20.7)$ & $19.1 \pm 0.3(16.4-22.7)$ \\
Tail length & $61.0 \pm 0.2(59.5-62.5)$ & $65.0 \pm 2.0(58.2-73.6)$ \\
$\quad \begin{array}{l}\text { Length of hyaline } \\
\quad \text { ail terminus }\end{array}$ & $16.9 \pm 0.2(13.6-17.8)$ & $14.6 \pm 0.6(10.9-16.4)$ \\
$\quad$ Excretory pore to & $91.5 \pm 0.4(86.9-95.2)$ & $82.7 \pm 4.0(74.5-105.5)$ \\
$\quad$ head end & & \\
Stylet length & $12.8 \pm 0.3(11.9-15.4)$ & $10.4 \pm 0.3(9.1-12.3)$ \\
Stylet knob height & $1.8 \pm 0.0(1.7-1.9)$ & $1.8 \pm 0.0(1.8-1.9)$ \\
Stylet knob width & $2.3 \pm 0.0(2.2-2.3)$ & $2.9 \pm 0.1(2.3-3.6)$ \\
DGO & $2.9 \pm 0.0(2.9-3.0)$ & $2.9 \pm 0.1(1.8-3.6)$ \\
Ratio a & $23.5 \pm 0.3(21.2-27.0)$ & $22.0 \pm 0.4(18.5-27.8)$ \\
Ratio c & $7.3 \pm 0.0(7.0-7.7)$ & $7.3 \pm 0.2(5.5-9.9)$ \\
\hline
\end{tabular}

${ }^{a}$ Twenty individuals were measured for each characteristic (means \pm standard error [SE]).

b Original description from Eisenback et al. (2003).
Center for Biotechnology Information (NCBI) databases were aligned over the same length in CLUSTALW and MUSCLE using MEGA v. 6 (Tamura et al. 2013). The evolutionary history was inferred by using the maximum-likelihood (ML) method based on the Tamura-Nei model (Tamura and Nei 1993). Initial trees for the heuristic search were obtained automatically by applying NeighborJoin and BioNJ algorithms to a matrix of pairwise distances estimated using the maximum composite likelihood approach, and then selecting the topology with superior log-likelihood value. Evolutionary analyses were conducted in MEGA v.6. Bootstrap analysis with 1,000 replicates was conducted to assess the degree of support for each branch on the tree. Unique sequence data obtained from the RKN in this study were submitted to GenBank at NCBI under accession number, KT783539, KU174206, and KU174207.

\section{Results}

Morphometric and morphological analysis. Morphometrics of M. haplanaria $\mathbf{J} 2(n=20)$ obtained from the field in Florida (FL isolate) was compared with the original $M$. haplanaria description by Eisenback et al. (2003) (Table 2). Most of the J2 morphological characteristics of the FL isolate of $M$. haplanaria were comparable with those reported in the original description. However, some of the morphological characteristics such as body length $(448.2 \pm 2.3 \mu \mathrm{m})$, stylet length $(12.8 \pm 0.3 \mu \mathrm{m})$, and tail hyaline length $(16.9 \pm 0.2 \mu \mathrm{m})$ showed higher values for the FL isolate than reported in the original description (Table 2). The perineal patterns of $M$. haplanaria showed the characteristic extremely rounded to oval shaped, as reported in the original description (Fig. 1).

Examining the specificity of primers used for RKN molecular diagnosis in discriminating M. haplanaria. According to Blok et al. (1997), the nontranscribed spacer region between the $5 \mathrm{~S}$ and $18 \mathrm{~S}$ rDNA genes is routinely amplified from single juveniles of all RKN with the 194/195 primers. The PCR amplification from single female using 194/195 primers gave amplification products of $720 \mathrm{bp}$ from the tropical species $M$. javanica (FL isolate), M. incognita (FL isolate), and $M$. arenaria (FL isolate); 700 bp from M. hapla (VW9 strain provided by Valerie Williamson, University of California, Davis); and $780 \mathrm{bp}$ from $M$. enterolobii (FL isolate).This set of primers failed to amplify the nontranscribed spacer region from M. haplanaria females (Fig. 2A). Four pairs of RKN-specific sequencecharacterized amplified region (SCAR) primers (Table 1) were tested with extracts from $M$. incognita, $M$. arenaria, $M$. javanica, $M$. hapla, M. enterolobii, and M. haplanaria. The PCR with specific SCAR primers for $M$. incognita, $M$. arenaria, $M$. javanica, and $M$. hapla resulted in the amplification of expected product size from corresponding
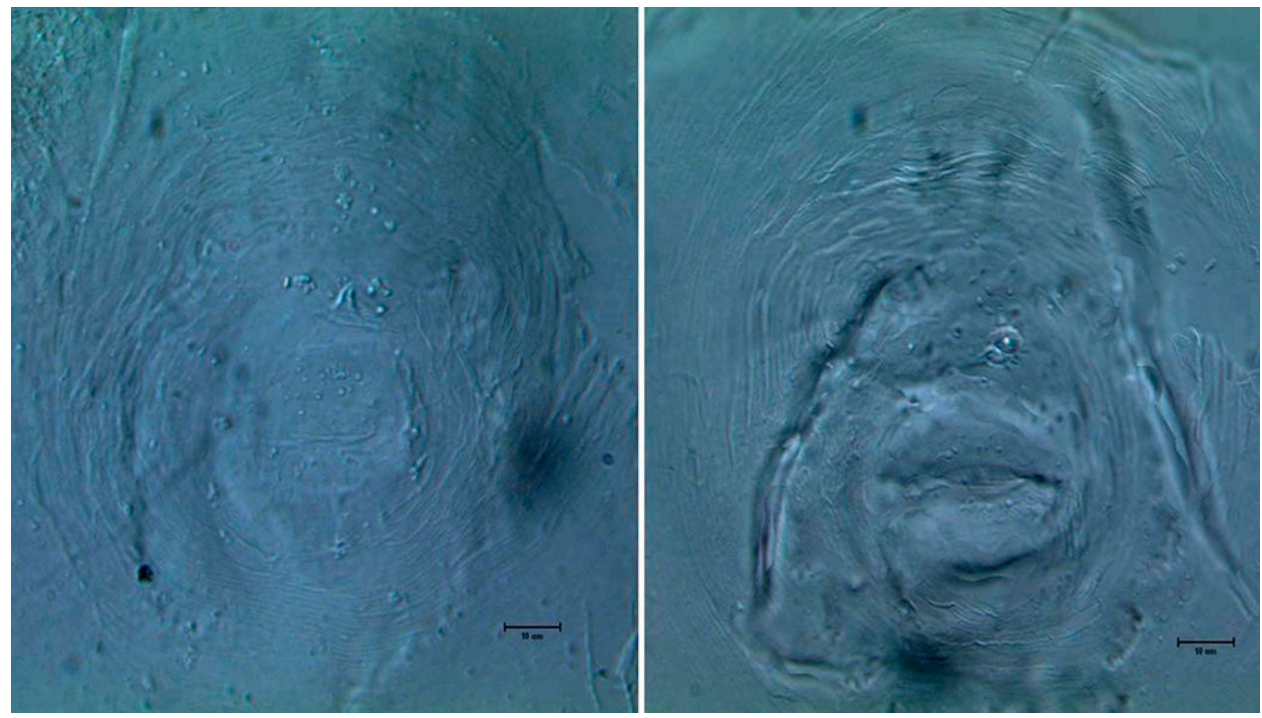

Fig. 1. Photomicrographs of perineal patterns of two individual females of Meloidogyne haplanaria from Florida. Scale bar $=10 \mu \mathrm{m}$. 
species (Meng et al. 2004; Wishart et al. 2002; Zijlstra et al. 2000) whereas no product was amplified from $M$. haplanaria (data not shown). The specificity of these primers was in accordance with previous reports (Adam et al. 2007; Powers et al. 2005). However, the PCR with rDNA-IGS2 internal primers Me-F/R to amplify a specific sequence from $M$. enterolobii resulted in the amplification of an approximately 200-bp product from both $M$. enterolobii and M. haplanaria (Fig. 2B).

The primers $63 \mathrm{VNL} / 63 \mathrm{VTH}$, targeting a 63 -bp tandem repeat region of the mtDNA, produced a 322-bp fragment from $M$. enterolobii and an approximately 800-bp fragment from $M$. incognita and $M$. javanica, whereas either approximately $500 \mathrm{bp}$ or multiple bands were obtained from M. arenaria and M. hapla, respectively (Brito et al. 2004; Lunt et al. 1998; Stanton et al. 1997). However, no amplification product was obtained from $M$. haplanaria with the 63VNL/63VTH primers (Fig. 3A). The PCR amplifications with primer set $\mathrm{C} 2 \mathrm{~F} 3 / 1108$ located in the mtDNA COII and $16 \mathrm{~S}$ ribosomal
mtDNA, respectively, produced a 540-bp amplicon from $M$. haplanaria whereas, for M. hapla, a 528-bp amplicon was produced. Amplification from $M$. incognita, $M$. javanica, and $M$. arenaria did not give any products, while $M$. enterolobii generated a unique product of $705 \mathrm{bp}$ (Fig. 3B). In addition to COII, an additional mtDNA marker from the COI region was amplified using JB3/CoI2RF primers on all six RKN species tested in this study. No variation in the product size (approximately $800 \mathrm{bp}$ ) was observed between the species (data not shown).

Sequence analysis and phylogenetic analysis. The BLAST analysis of the 540-bp fragment between mtDNA COII and 16S ribosomal gene sequences from four individual adult females revealed 99\% identity with the $M$. haplanaria sequence of isolate AnM6 from Texas (GenBank accession number AY757905) across the entire $540 \mathrm{bp}$ of the fragment (100\% query cover) (Powers et al. 2005). The sequence of the FL isolate of $M$. haplanaria differed from that of the Texas isolate by a single nucleotide at the base position, 209 (A/T).

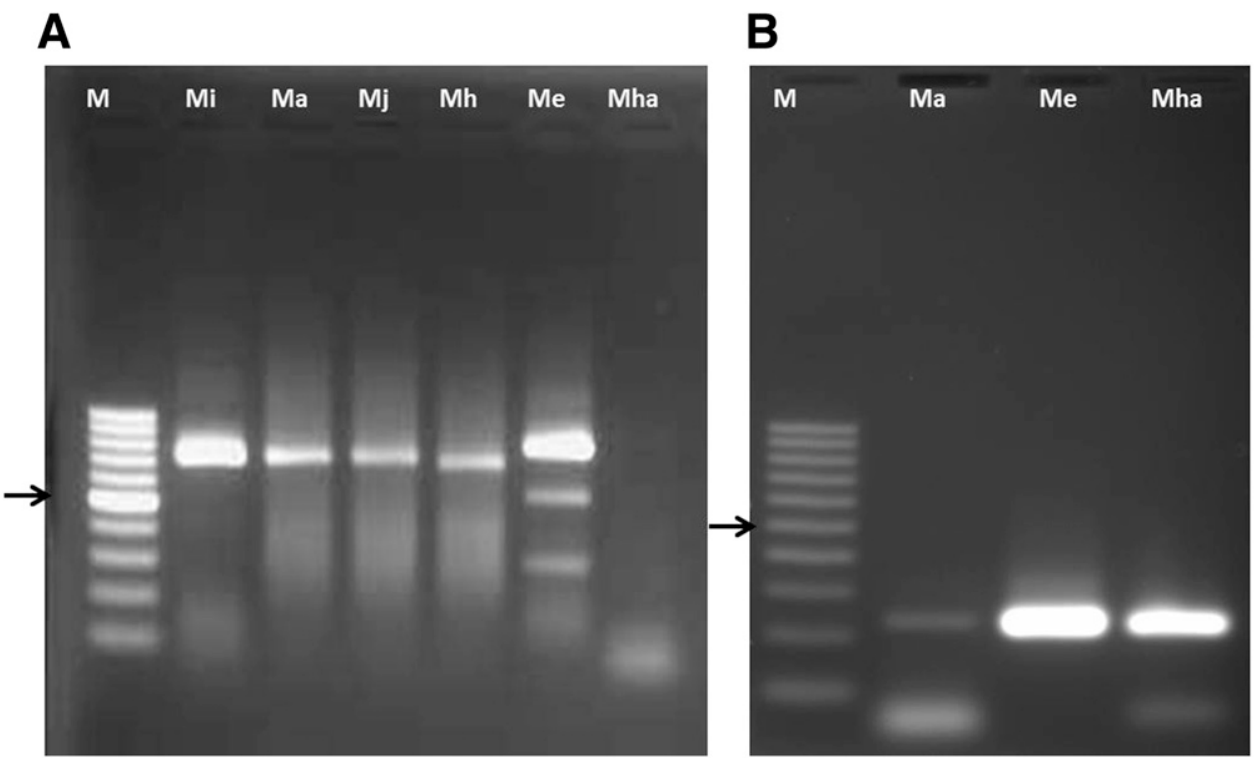

Fig. 2. A, Amplification of the nontranscribed spacer region between the $5 S$ and $18 S$ rDNA using 194/195 primers. B, Polymerase chain reaction amplification with rDNA-IGS2 internal primers Me-F/R to amplify specific sequence from Meloidogyne enterolobii. Species of DNA sources are indicated above each lane: $M i=M$. incognita, $M a=M$. arenaria, $M j=$ M. javanica, $\mathrm{Mh}=\mathrm{M}$. hapla, $\mathrm{Me}=\mathrm{M}$. enterolobii, and $\mathrm{Mha}=\mathrm{M}$. haplanaria. Lane $\mathrm{M}=100$-bp marker ladder (Fermentas), with the position of the 500-bp band indicated by the arrows.
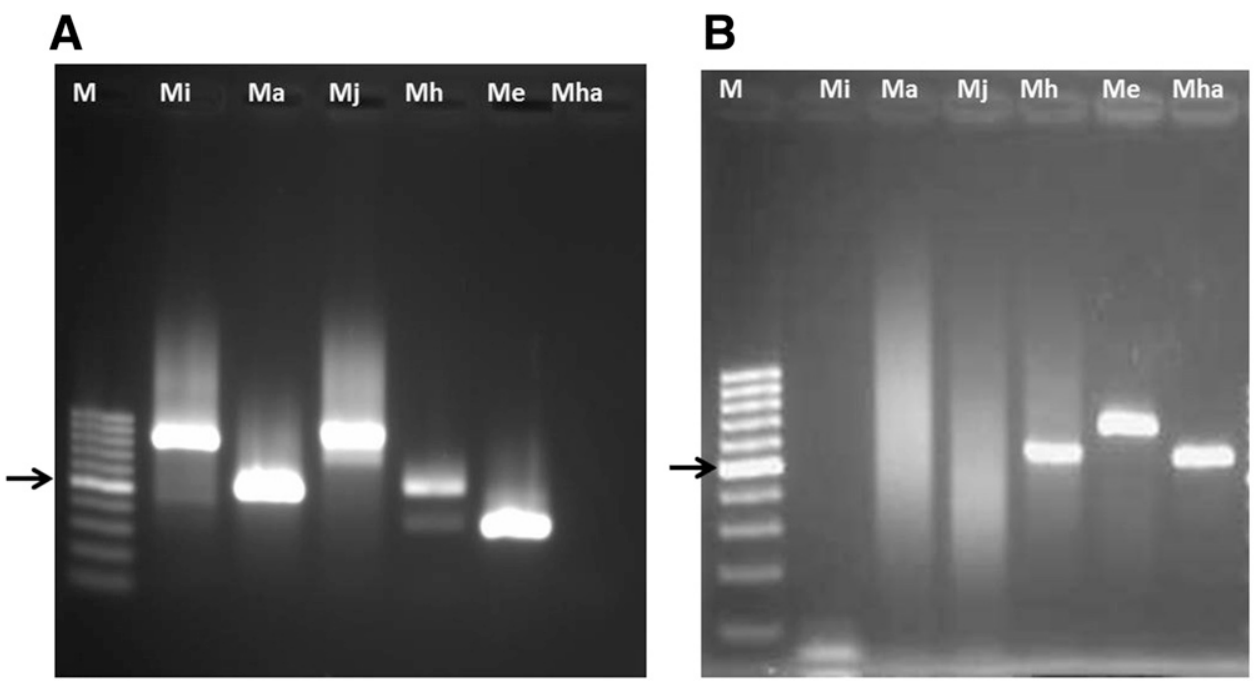

Fig. 3. A, Amplification of a 63-bp tandem repeat region of the mitochondrial genome using 63VNL/63VTH primers. B, Amplification of mitochondrial DNA region including COII and $16 \mathrm{~S}$ rRNA genes using C2F3 and 1108 primers. Species of DNA sources are indicated above each lane: $\mathrm{Mi}=$ Meloidogyne incognita, $\mathrm{Ma}=\mathrm{M}$. arenaria, $\mathrm{Mj}=\mathrm{M}$. javanica, $\mathrm{Mh}=$ M. hapla, $\mathrm{Me}=\mathrm{M}$. enterolobii, and Mha $=$ M. haplanaria. Lane $\mathrm{M}=100$-bp marker ladder (Fermentas), with the position of the 500-bp band indicated by the arrows. 
M. haplanaria shared $90 \%$ similarity (80\% query cover) with $M$. javanica (GenBank accession number L76261). Further sequence examination revealed that the $M$. haplanaria sequence has a deletion of 1,097 bp that included part of the COII region ( $378 \mathrm{bp}$ ) and the IGS region (719 bp between the primer sets MORF and MTHIS), as compared with $M$. javanica sequence (1,640 bp). M. haplanaria showed $87 \%$ similarity ( $87 \%$ query cover) with the $M$. enterolobii sequence (GenBank accession number KF993632), and further sequence comparison between $M$. haplanaria and $M$. enterolobii showed that both sequences lacked parts of the COII region and the IGS region as compared with M. javanica. However, M. enterolobii has an insertion of $166 \mathrm{bp}$ adjacent to primer TRNAH. The sequence alignment of M. hapla (GenBank accession number AY942850) and M. haplanaria revealed that parts of the COII and IGS regions were also absent in both nematodes; however, sequences of both M. hapla (528 bp) and M. haplanaria (540 bp) shared only $81 \%$ similarity ( $100 \%$ query cover).

In addition to the COII gene, the mtDNA COI gene has also been used as DNA barcoding loci in distinguishing RKN species (Kiewnick et al. 2014). However, no sequence information is currently available in the database for the COI gene from M. haplanaria. The BLAST analysis of COI fragments from $M$. haplanaria showed $90 \%$ identity with the COI region of $M$. incognita from KP202350, $M$. javanica from KP202352, and $M$. arenaria from KP202350, while there was $89 \%$ identity with $M$. enterolobii from KP202351. Alignment and phylogenetic analysis of COII sequences revealed several clades which were separated by varying bootstrap support values in the ML analysis (Fig. 4). The optimal phylogenetic tree obtained from the ML analysis revealed that the four sequences from the FL isolate of $M$. haplanaria and the M. haplanaria sequence retrieved from GenBank formed a monophyletic group with a high bootstrap value of $100 \%$. All RKN formed two well-supported clades (93 and 100\%), with the exclusion of $M$. graminis and $M$. marylandi, which were grouped together with $100 \%$ bootstrap support (Fig. 4).

Determination of mitochondrial haplotype of $M$. haplanaria. The mitochondrial primer set developed by Powers and Harris (1993) did not generate any products from $M$. incognita, $M$. javanica, and $M$. arenaria. The size difference in the amplification products of C2F3/1108 from M. haplanaria (540 bp) and M. hapla (528 bp) was too small to discriminate these species from each other just by the gel visualization. In this study, we used the mtDNA assay of Pagan et al. (2014), using the primer set developed by Stanton et al. (1997) that was successful in resolving Meloidogyne spp. Primers TRNAH and MRH106, which amplify a region of the mtDNA including part of lrDNA gene, amplified a fragment of approximately $557 \mathrm{bp}$ from all of the RKN tested, except for $M$. enterolobii, which produced a fragment of approximately 722 bp (Fig. 5A). Primers MORF and MTHIS, which amplify the mtDNA IGS region, did not amplify any product from $M$. hapla, M. enterolobii, and M. haplanaria. The sequence analysis has also showed that the sequence fragment in the IGS region between the primer sets MORF and MTHIS was deleted in $M$. haplanaria, M. hapla, and M. enterolobii. A fragment of approximately $743 \mathrm{bp}$ was amplified from $M$. incognita and $M$. javanica whereas the product from $M$. arenaria was only 214 bp in length (Fig. 5B). Based on the amplification products by the two primer sets, M. haplanaria was not differentiated from M. hapla. However, based on the sizes of the MnlI and HinfI digestion products

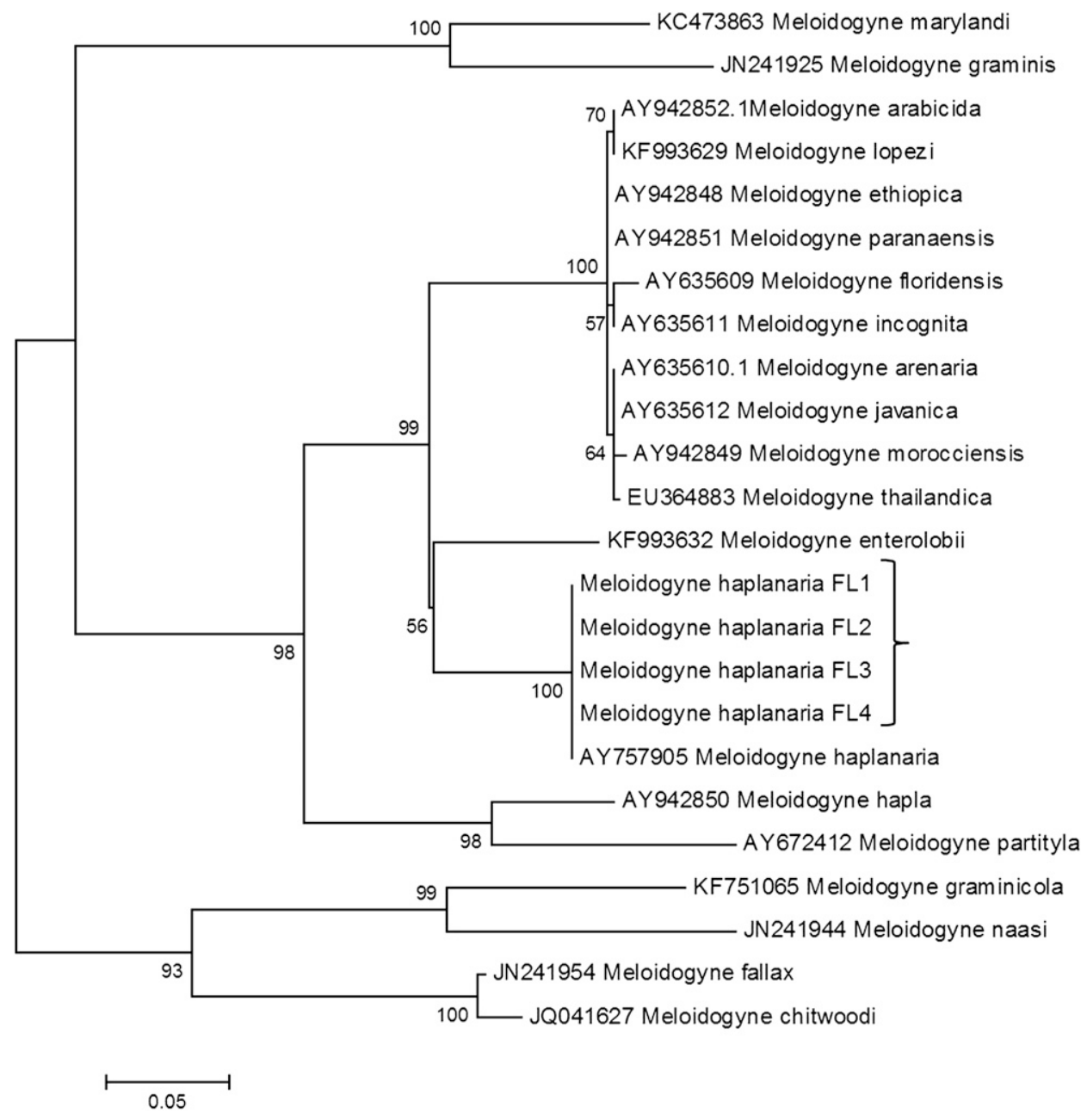

Fig. 4. Maximum-likelihood tree after an alignment of COII-IrDNA region of the mitochondrial genome of Meloidogyne populations. The four individual sequences from the Florida isolates of Meloidogyne haplanaria formed a monophyletic group with the sequence of M. haplanaria from Texas (AY757905). Bootstrap support for each clade is indicated at the nodes. 
of the TRNAH/MRH106 fragments and the presence and size of the MORF/MTHIS fragment, we determined the mitochondrial haplotype of $M$. haplanaria, which gave a clear separation from $M$. hapla and other tropical RKN species (Fig. 6). Although both M. hapla and M. haplanaria gave $557 \mathrm{bp}$ of TRNAH/MRH106 fragments, the amplification with the primer set MORF and MTHIS did not yield any products in both species. However, the digestion assay of TRNAH/MRH106 using HinfI did not show any digestion products in M. haplanaria whereas two digestion products of 446 and $110 \mathrm{bp}$ were present in the case of $M$. hapla. The digestion assay with $M n l I$ did not yield any product in $M$. hapla but yielded two digestion products of 416 and $140 \mathrm{bp}$ in M. haplanaria (Table 3).

All samples of $M$. haplanaria (single females and juveniles) gave the same haplotype pattern for the TRNAH/MRH106 fragment following digestion with the MnlI and HinfI (Fig. 7). The exact size of the restriction fragments by each enzyme from all the RKN were estimated using Restriction Mapper v. 3 (Table 3). The restriction pattern revealed a distinct haplotype for $M$. haplanaria, which is easily distinguished from other RKN species.

\section{Discussion}

Nematode identification based on molecular approaches is becoming widely accepted due to the relative simplicity of its application. Molecular approaches provide accuracy, speed, reliability, and affordability in nematode identification as well as enable characterization of new nematode species. The diagnosis strategy based on mtDNA analysis has been useful to identify and address unforeseen plantparasitic nematode threats to agricultural crops. This approach has been utilized to distinguish morphologically similar but genetically divergent nematode species as well as new and emerging nematode parasites. Pagan et al. (2014) demonstrated that, even though the differences between the mtDNA of Meloidogyne spp. are limited, these differences align with other identification criteria based on esterase phenotype. Thus, the mtDNA haplotype approach can be the first screening step to assess the variability of RKN species in areas that have not yet been well surveyed. In our molecular study, the haplotype pattern of $M$. haplanaria did not match with any of the haplotype patterns that has been reported thus far (Pagan et al. 2015; Powers and Harris 1993; Powers et al. 1986). Further morphological and sequence analysis based on the mtDNA sequence that span, together, the spacer region and part of the lrDNA gene also confirmed that the RKN species isolated from the $M i$-resistant tomato plants from Naples, FL was M. haplanaria, the Texas peanut RKN. The morphometrics of $\mathrm{J} 2$ of $M$. haplanaria showed that the stylet length and hyaline tail length of the FL isolate had higher lower-range values than the original description. However, lengths of both the hyaline tail and stylet were greater in $M$. haplanaria than other RKN species, as reported by Eisenback et al. (2003). In our study, we observed less variation in morphological measurements compared with the original description. The morphology of the perineal patterns is considered to be one of the most important morphological characteristic for RKN species identification (Eisenback 1985; Karssen and

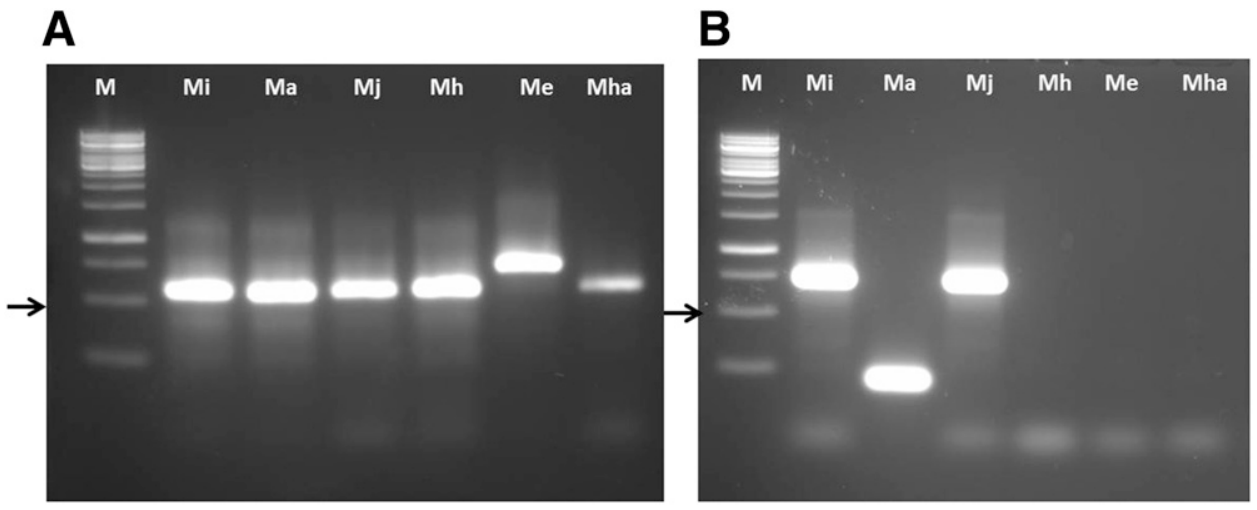

Fig. 5. A, Amplification products from characterized Meloidogyne spp. populations obtained using primers TRNAH and MRH106. B, Amplification obtained using primers MORF and MTHIS. Species of DNA sources are indicated above each lane: $\mathrm{Mi}=$ Meloidogyne incognita, $M a=M$. arenaria, $\mathrm{Mj}=\mathrm{M}$. javanica, $\mathrm{Mh}=\mathrm{M}$. hapla, $\mathrm{Me}=\mathrm{M}$. enterolobii, and Mha $=M$. haplanaria. Lane $\mathrm{M}=1$-kb marker ladder (Fermentas), with the position of the 500-bp band indicated by the arrows.

\section{A}

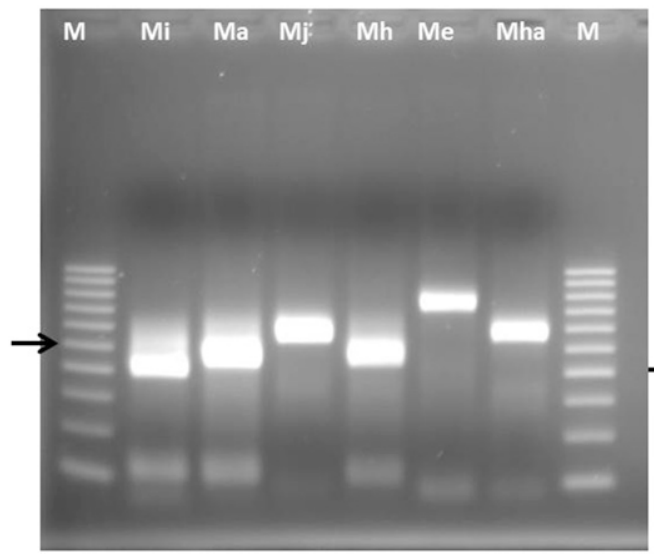

B

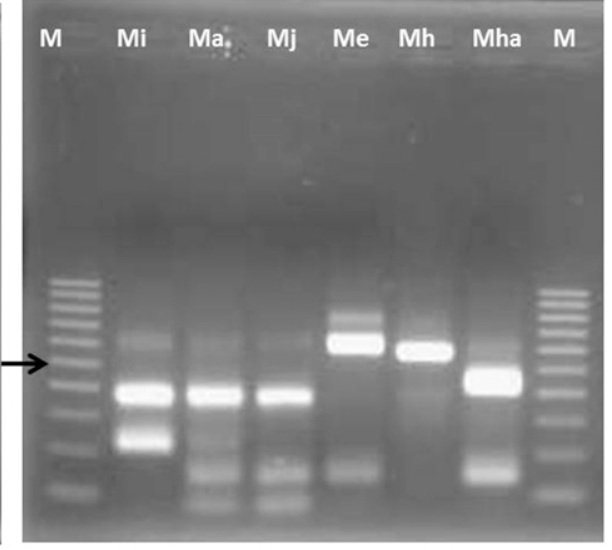

Fig. 6. Restriction fragments obtained after cleavage of the amplification products using TRNAH/MRH106 with A, Hinfl or B, Mnll. Species of DNA sources are indicated above each lane: $\mathrm{Mi}=$ Meloidogyne incognita, $\mathrm{Ma}=\mathrm{M}$. arenaria, $\mathrm{Mj}=\mathrm{M}$. javanica, $\mathrm{Mh}=\mathrm{M}$. hapla, $\mathrm{Me}=\mathrm{M}$. enterolobii, and Mha = M. haplanaria. Lane $\mathrm{M}=100$-bp marker ladder (Fermentas), with the position of the 500-bp band indicated by the arrows. 
van Aelst 2001). The perineal pattern of adult females was extremely round to oval shaped, as in the original description (Eisenback et al. 2003).

Molecular diagnostics based on mtDNA has increasingly been used for RKN identification at species level and also for constructing phylogenies (Blok and Powers 2009). The COII and 16s rRNA gene regions of the mtDNA show length polymorphism due to an AT-rich noncoding sequence with different sizes, as a result of deletions and insertions (Blok and Powers 2009). Eisenback et al. (2003) considered $M$. haplanaria to be a distinct species from other RKN such as $M$. chitwoodi, M. fallax, M. graminis, M. hapla, M. arenaria, $M$. incognita, and $M$. javanica. The ML analysis of COII-lrDNA mtDNA region grouped $M$. haplanaria and $M$. enterolobii together but separate from the other RKN species mentioned above. Considering the main clades, our results also agree with those obtained by García and SánchezPuerta (2015) for the cox2-rrnS mtDNA region.

In this study, we also examined the specificity of primers that are routinely used for RKN diagnosis in distinguishing $M$. haplanaria from other RKN species. Adam et al. (2007) suggested a molecular tool to distinguish the seven most common and economically important Meloidogyne spp., based on the PCR amplification products using 194/195 primer sets (Blok et al. 1997), which amplifies a nontranscribed spacer region between the 5S and 18S rDNA genes. The PCR using the aforementioned primer set did not produce any product from M. haplanaria whereas other RKN species gave unique products. The result was consistent with all of the samples of $M$. haplanaria tested in this study. Similarly, primer set $63 \mathrm{VNL} / 63 \mathrm{VTH}$, targeting a 63-bp tandem repeat region of the mtDNA gave a unique product size of $322 \mathrm{bp}$ from M. enterolobii, whereas no product was amplified from M. haplanaria. Although the 63-bp tandem repeat region has been used to differentiate and characterize Meloidogyne spp., it has been shown that $M$. javanica, $M$. arenaria, and $M$. incognita exhibit hypervariability in this region (Blok et al. 2002). Thus, the 63-bp tandem repeat region of the mtDNA is not a suitable marker to distinguish $M$. haplanaria from these RKN species. The rDNA-IGS2 internal primers (Me-F/R) (Hu et al. 2011) used to identify M. enterolobii gave the same product size in both the FL isolate of $M$. haplanaria and $M$. enetrolobii. However, the PCR amplifications with primer set $\mathrm{C} 2 \mathrm{~F} 3 / 1108$ of the COII and $16 \mathrm{~S}$ ribosomal mtDNA, respectively, produced a 540-bp amplicon from $M$. haplanaria while the amplification from $M$. enterolobii gave a unique product of $705 \mathrm{bp}$. The sequence alignment between these two species revealed an insertion of $166 \mathrm{bp}$ in M. enterolobii, which clearly indicates that the FL isolate is different from $M$. enterolobii. Therefore, it could be concluded that $\mathrm{Me}-\mathrm{F} / \mathrm{R}$ primers are not specific to $M$. enterolobii.

The phylogenetic analysis based on the COII-lrDNA region showed that $M$. haplanaria is closely related to $M$. enterolobii. The M. enterolobii-specific primers ( $\mathrm{Hu}$ et al. 2011) did not differentiate M. enterolobii from $M$. haplanaria, and this may lead to misidentification of these nematode species. Although the amplification of the mtDNA region that, together, spans the spacer region and part of lrDNA gene using the primer set $\mathrm{C} 2 \mathrm{~F} 3 / 1108$, has been used as a reliable diagnostic marker to distinguish major $\mathrm{RKN}$ species, this primer set failed to amplify any product from $M$. incognita, $M$. javanica, and $M$. arenaria in our study, and it was also unable to differentiate $M$. hapla from $M$. haplanaria based on the size of the amplification product. The absence of the product may indicate either a failed reaction or a new species or variant; hence, the RKN diagnosis based on length polymorphism using primer set $\mathrm{C} 2 \mathrm{~F} 3 / 1108$ may not always be suitable for differentiating major RKN species. Thus, the examination of more than one molecular characteristic will be a suitable approach for identification and evolutionary studies of a particular species. In our study, the diagnostic strategy suggested by Pagan et al. (2014) gave the best resolution in discriminating $M$. haplanaria from other important $\mathrm{RKN}$ species that can reproduce on $M i$-resistant tomato plants, especially M. hapla and M. enterolobii. Additionally, this diagnostic strategy will be faster and cheaper than proceeding directly to sequencing.

The current distribution of M. haplanaria in the United States is limited to peanut fields in Texas and Arkansas. Our study confirms the presence of this nematode in Florida, which poses a potential threat to peanut and tomato production in the state. The $M$. haplanaria

Table 3. Polymerase chain reaction amplification profiles yielded by TRNAH/MRH106 and MORF/MTHIS primers and restriction fragment length polymorphism products yielded by Hinfl and MnlI digestion restriction enzymes

\begin{tabular}{lcccc}
\hline & \multicolumn{4}{c}{ TRNAH/MRH106 fragment (bp) } \\
\cline { 2 - 4 } Meloidogyne spp. & Fragment & HinfI digestion & MnlI digestion & MORF/MTHIS \\
\hline Meloidogyne incognita & 557 & $396,112,49$ & 340,217 \\
M. arenaria & 557 & 445,112 & $340,140,77$ & 742 \\
M. javanica & 558 & 558 & $341,140,77$ & 214 \\
M. enterolobii & 723 & 723 & 583,140 & 743 \\
M. hapla & 556 & 446,110 & 556 & $\mathrm{NP}$ \\
M. haplanaria & 556 & 556 & 416,140 & $\mathrm{NP}$ \\
\hline
\end{tabular}

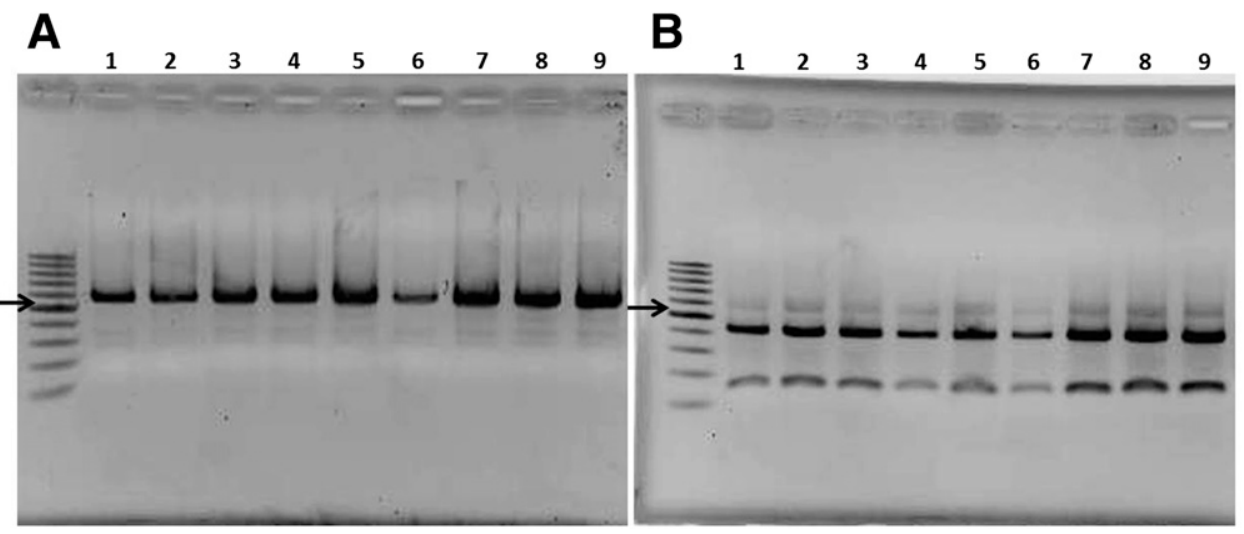

Fig. 7. Restriction fragments obtained after cleavage of the amplification products using TRNAH/MRH106 with A, Hinfl or B, Mnll from nine samples of Meloidogyne haplanaria (FL isolate). Lanes 1 to 8 represent samples from gravid females and lane 9 represents the sample from juveniles extracted from original field soil sample. Lane $\mathrm{M}=100$-bp marker ladder (Fermentas), with the position of the $500-\mathrm{bp}$ band indicated by the arrows. 
infestation in tomato fields in Naples, FL produced heavy galling and severe yield reduction (data not shown). Currently available $\mathrm{Mi}$ resistant tomato varieties and rootstocks may not be effective against $M$. haplanaria. Therefore, there is a need to screen different tomato varieties for $M$. haplanaria resistance in order to select varieties suitable for integrated management programs. A reliable and rapid identification of M. haplanaria in vegetable fields in Florida is of paramount importance for containing, monitoring, and managing the spread and damage caused by this nematode.

\section{Acknowledgments}

We thank the United States Department of Agriculture National Institute of Food and Agriculture for providing the financial support for the study and V. Williamson, University of California, Davis for providing M. hapla VW9 isolates.

\section{Literature Cited}

Adam, M. A. M., Phillips, M. S., and Blok, V. C. 2007. Molecular diagnostic key for identification of single juveniles of seven common and economically important species of root-knot nematode (Meloidogyne spp.). Plant Pathol. 56:190-197.

Bendezu, F., Morgan, E., and Starr, J. L. 2004. Hosts for Meloidogyne haplanaria. Nematropica 34:205-209.

Benson, D. A., Cavanaugh, M., Clark, K., Karsch-Mizrachi, I., Lipman, D. J., Ostell, J., and Sayers, E. W. 2013. GenBank. Nucleic Acids Res. 41:D36-D42.

Blok, V., Phillips, M., and Fargette, M. 1997. Comparison of sequences from the ribosomal DNA intergenic region of Meloidogyne mayaguensis and other major tropical root-knot nematodes. J. Nematol. 29:16-22.

Blok, V. C., and Powers, T. O. 2009. Biochemical and molecular identification. Pages 98-118 in: Root-knot Nematodes. R. N. Perry, M. Moens, and J. Starr, eds. CABI Publishing, Wallingford, UK.

Blok, V. C., Wishart, J., Fargette, M., Berthier, K., and Phillips, M. S. 2002. Mitochondrial DNA differences distinguishing Meloidogyne mayaguensis from the major species of tropical root-knot nematodes. Nematology 4: 773-781.

Brito, J., Powers, T. O., Mullin, P. G., Inserra, R. N., and Dickson, D. W. 2004. Morphological and molecular characterization of Meloidogyne mayaguensis isolates from Florida J. Nematology 36:232-240.

Churamani, R., McGawley, R. T., and Overstreet, E. C. 2015. Meloidogyne spp. reported from Arkansas: Past and Present. Page 60 in: (Abstr.) Proc. 54th Annu. Meet. Soc. Nematol. East Lansing, MI

Eisenback, J. D. 1985. Diagnostic characters useful in the identification of the four most common species of root-knot nematodes (Meloidogyne spp.). Pages 95-112 in: An Advanced Treatise on Meloidogyne, Vol. I, Biology and Control. J. N. Sasser and C. C. Carter, eds. North Carolina State University Graphics, Raleigh.

Eisenback, J. D., Bernard, E. C., Starr, J. L., Lee, T. A., and Tomaszewski, E. K. 2003. Meloidogyne haplanaria $\mathrm{n}$. sp. (Nematoda: Meloidogynidae), a root-knot nematode parasitizing peanut in Texas. J. Nematol. 35:395-403.

Esser, R. P. 1986. A water agar en face technique. Proc. Helminthol. Soc. Wash. 53:254-255.

Esser, R. P., Perry, V. G., and Taylor, A. L. 1976. A diagnostic compendium of the genus Meloidogyne (Nematoda: Heteroderidae). Proc. Helminthol. Soc. Wash. 43:138-150.

FDACS. 2012. Florida Agriculture Overview and Statistics. Online publication. Florida Department of Agriculture and Consumer Services (FDACS). http://www. freshfromflorida.com/Divisions-Offices/Marketing-and-Development/ Education/For-Researchers/Florida-Agriculture-Overview-and-Statistics

García, L. E., and Sánchez-Puerta, M. V. 2015. Comparative and evolutionary analyses of Meloidogyne spp. based on mitochondrial genome sequences. PLoS One 10:e0121142.
Hall, T. A. 1999. BioEdit: A user-friendly biological sequence alignment editor and analysis program for Windows 95/98/NT. Nucleic Acids Symp. Ser. 41:95-98.

Harrison, J. M., and Green, C. D. 1976. Comparison of centrifugal and other methods for standardization of extraction of nematodes from soil. Ann. Appl. Biol. 82:299-308.

Hartman, K. M., and Sasser, J. N. 1985. Identification of Meloidogyne species on the basis of differential host test and perineal patterns morphology. Pages 69-77 in: An Advanced Treatise on Meloidogyne, Vol. II, Methodology. K. R. Barker, C. C. Carter, and J. N. Sasser, eds. North Carolina State University Graphics, Raleigh.

Hu, M. X., Zhuo, K., and Liao, J. L. 2011. Multiplex PCR for the simultaneous identification and detection of Meloidogyne incognita, M. enterolobii, and $M$. javanica using DNA extracted directly from individual galls. Phytopathology 101:1270-1277.

Karssen, G., and van Aelst, A. 2001. Root-knot nematode perineal pattern development: A reconsideration. Nematology 3:95-111.

Kiewnick, S., Holterman, M., van den Elsen, S., van Megen, H., Frey, J., and Helder, J. 2014. Comparison of two short DNA barcoding loci (COI and COII) and two longer ribosomal DNA genes (SSU \& LSU rRNA) for specimen identification among quarantine root-knot nematodes (Meloidogyne spp.) and their close relatives. Eur. J. Plant Pathol. 140:97-110.

Lunt, D. H., Whipple, L. E., and Hyman, B. C. 1998. Mitochondrial DNA variable number tandem repeats (VNTRs): Utility and problems in molecular ecology. Mol. Ecol. 7:1441-1455.

Meng, Q., Long, H., and Xu, J. 2004. PCR assays for rapid and sensitive identification of three major root-knot nematodes, Meloidogyne incognita, M. javanica and M. arenaria. Acta Phytopathol. Sin. 34:204-210.

Pagan, C., Coyne, D., Carneiro, R., Kariuki, G., Luambano, N., Affokpon, A., and Williamson, V. M. 2015. Mitochondrial haplotype-based identification of ethanol-preserved root-knot nematodes from Africa. Phytopathology 105: 350-357.

Powers, T. O., and Harris, T. S. 1993. A polymerase chain reaction method for identification of five major Meloidogyne spp. J. Nematol. 25:1-6.

Powers, T. O., Mullin, P. G., Harris, T. S., Sutton, L. A., and Higgins, R. S. 2005 Incorporating molecular identification of Meloidogyne spp. into a large-scale regional nematode survey. J. Nematol. 37:226-235.

Powers, T. O., Platzer, E. G., and Hyman, B. C. 1986. Species-specific restriction site polymorphism in root-knot nematode mitochondrial DNA. J. Nematol. 18: 288-293.

Simpson, C. E., Starr, J. L., Church, G. T., Burow, M. D., and Paterson, A. H. 2003. Registration of 'NemaTAM' peanut. Crop Sci. 43:1561.

Stanton, J., Hugall, A., and Moritz, C. 1997. Nucleotide polymorphisms and an improved PCR-based mtDNA diagnostic for parthenogenetic root-knot nematodes (Meloidogyne spp.). Fundam. Appl. Nematol. 20:261-268.

Tamura, K., and Nei, M. 1993. Estimation of the number of nucleotide substitutions in the control region of mitochondrial DNA in humans and chimpanzees. Mol. Biol. Evol. 10:512-526.

Tamura, K., Stecher, G., Peterson, D., Filipski, A., and Kumar, S. 2013. MEGA6: Molecular Evolutionary Genetics Analysis Version 6.0. Mol. Biol. Evol. 30: $2725-2729$

Trudgill, D. L., and Blok, V. C. 2001. Apomictic polyphagous root knot nematodes Exceptionally successful and damaging biotrophic root pathogens. Annu. Rev. Phytopathol. 39:53-77.

Williamson, V. M. 1998. Root-knot resistance genes in tomato and their potential for future use. Annu. Rev. Phytopathol. 36:277-293.

Wishart, J., Philips, M. S., and Blok, V. C. 2002. Ribosomal intergenic spacer A polymerase chain reaction diagnostic for Meloidogyne chitwoodi, M. fallax, and $M$. hapla. Phytopathology 92:884-892.

Zijlstra, C., Donkers-Venne, D. T., and Fargette, M. 2000. Identification of Meloidogyne incognita, $M$. javanica and $M$. arenaria using sequence characterized amplified region (SCAR) based PCR assays. Nematology 2:847-853. 